\title{
The Chromatic Number of the $q$-Kneser Graph for Large $q$
}

\author{
Ferdinand Ihringer* \\ Department of Mathematics: \\ Analysis, Logic and Discrete Mathematics. \\ Ghent University, Belgium.
}

Submitted: Jun 1, 2018; Accepted: Feb 28, 2019; Published: Mar 22, 2019

(C) The authors. Released under the CC BY-ND license (International 4.0).

\begin{abstract}
We obtain a new weak Hilton-Milner type result for intersecting families of $k$ spaces in $\mathbb{F}_{q}^{2 k}$, which improves several known results. In particular the chromatic number of the $q$-Kneser graph $q K_{n: k}$ was previously known for $n>2 k$ (except for $n=2 k+1$ and $q=2)$ or $k<q \log q-q$. Our result determines the chromatic number of $q K_{2 k: k}$ for $q \geqslant 5$, so that the only remaining open cases are $(n, k)=(2 k, k)$ with $q \in\{2,3,4\}$ and $(n, k)=(2 k+1, k)$ with $q=2$.
\end{abstract}

Mathematics Subject Classifications: 51E20, 05C69, 05B25, 05D05, 06E30

\section{Introduction}

The Kneser graph $K_{n: k}$ has all $k$-sets of $\{1,2, \ldots, n\}, n \geqslant 2 k$, as vertices and two sets are adjacent if they are disjoint. The following conjecture due to Kneser [14] was shown by Lovász [15]:

Theorem 1 (Lovász (1978)). The chromatic number of $K_{n: k}$ is $n-2 k+2$.

We want to point out that the case $n=2 k$ is trivial as $K_{2 k: k}$ is bipartite. A natural generalization is the $q$-analog of the Kneser graph: the $q$-Kneser graph $q K_{n: k}$. Here we take the $k$-spaces of $\mathbb{F}_{q}^{n}$ as vertices and two vertices are adjacent if they intersect trivially. Let $\left[\begin{array}{l}n \\ k\end{array}\right]$ denote the number of $k$-spaces in $\mathbb{F}_{q}^{n}$. Note that for $0 \leqslant k \leqslant n$ we have

$$
\left[\begin{array}{l}
n \\
k
\end{array}\right]=\prod_{i=1}^{k} \frac{q^{k-i+1}-1}{q^{i}-1} .
$$

Due to two previous results by Blokhuis et al. [1] for $n>2 k$ and Blokhuis et al. [2] for $n=2 k$, we know the following:

\footnotetext{
*The author is supported by a postdoctoral fellowship of the Research Foundation — Flanders (FWO).
} 
Theorem 2 (Blokhuis, Brouwer, Chowdhury, Frankl, Mussche, Patkós, Szőnyi (2010), Blokhuis, Brouwer, Szönyi (2012)). If $k \geqslant 3$ and either $q \geqslant 3$ and $n \geqslant 2 k+1$, or $q=2$ and $n \geqslant 2 k+2$, then the chromatic number of $q K_{n: k}$ is $\left[^{n-k+1} \begin{array}{c}1 \\ 1\end{array}\right]$. If either $k<q \log q-q$ or $k \leqslant 3$, then the chromatic number of $q K_{2 k: k}$ is $q^{k}+q^{k-1}$.

We complete this result for $n=2 k$ and $q \geqslant 5$.

Theorem 3. Let $q \geqslant 5$. Then the chromatic number of $q K_{2 k: k}$ is $q^{k}+q^{k-1}$ for $n=2 k$.

The key ingredient of the $n=2 k$ proof by Blokhuis et al. [2] is a weak Hilton-Milner type result (see [11] for the Hilton-Milner theorem for the classical set case, a variation of the famous Erdős-Ko-Rado theorem [8]). In the following, we use projective notation, so we call 1-spaces points, 2-spaces lines, and $(n-1)$-spaces hyperplanes. Call the set of all $k$-spaces on fixed point a dictator (also known as point-pencil). The dual of a dictator consists of all $k$-spaces in a fixed hyperplane. Due to work by Hsieh [12], Frankl and Wilson [10], and Godsil and Newman [17], we know that the largest independent sets of $q K_{n: k}$ are dictators and, if $n=2 k$, duals of dictators, that is the family of all $k$-spaces in a hyperplane. Blokhuis et al. showed the following:

Theorem 4 (Blokhuis, Brouwer, Szőnyi (2011)). Let $k<q \log q-q$ and let $Y$ be an independent set of $q K_{2 k: k}$. If $Y$ is not contained in a dictator or its dual, then $|Y|<$ $q^{k(k-1)} / 2$.

Using a result by Tokushige on cross-intersecting families in vector spaces [20] and some properties of the spectrum of the Grassmann scheme, we improve this as follows:

Theorem 5. Let $q \geqslant 3$ and $k \geqslant 5$ and let $Y$ be an independent set of $q K_{2 k: k}$. If $Y$ is not contained in a dictator or its dual, then

$$
|Y|<\left(1+3 q^{-1}\right)\left[\begin{array}{c}
k \\
1
\end{array}\right]\left[\begin{array}{c}
2 k-2 \\
k-2
\end{array}\right] .
$$

As this does not cover $k=4$, we also provide the following:

Theorem 6. Let $q \geqslant 4$ and let $Y$ be an independent set of $q K_{2 k: k}$. If $Y$ is not contained in a dictator or its dual, then

$$
|Y|<3\left[\begin{array}{c}
k \\
1
\end{array}\right]\left[\begin{array}{c}
2 k-2 \\
k-2
\end{array}\right]
$$

While our second bound is slightly worse than Theorem 4 for $k$ and $q$ large, it is clearly better for $q$ small compared to $k$. It is easy to construct independent sets of $k$-spaces of size vaguely $\left[\begin{array}{c}k \\ 1\end{array}\right]\left[\begin{array}{c}2 k-2 \\ k-2\end{array}\right]$, so our result is close to a proper stability result.

Recently, Cameron-Liebler $k$-space classes (also known as Boolean degree 1 functions) received some attention $[3,9,18]$. In particular, Metsch showed the following [16]:

Theorem 7 (Metsch (2017)). Let $q \geqslant q_{0}$ for some universal constant $q_{0}$ and let $k<$ $q \log q-q-1$. Let $Y$ be a non-trivial Boolean degree 1 function on $k$-spaces of $\mathbb{F}_{q}^{2 k}$, then $|Y| \geqslant \frac{q}{5}\left[\begin{array}{c}2 k-1 \\ k-1\end{array}\right]$. 
The condition on $k$ comes from Theorem 4 , so with Theorem 5 we can improve this to the following:

Theorem 8. Let $q \geqslant q_{0}$ for some universal constant $q_{0}$. Let $Y$ be a non-trivial Boolean degree 1 function on $k$-spaces of $\mathbb{F}_{q}^{2 k}$, then $|Y| \geqslant \frac{q}{5}\left[\begin{array}{c}2 k-1 \\ k-1\end{array}\right]$.

Note that a tedious calculation shows that we can choose $q_{0}=89$ if we follow the argument in [16] without optimizing any of the used constants. We believe that all Boolean degree 1 functions for $k>2$ are trivial, so most likely might be still far from the truth.

Our paper is organized as follows: In Section 2, we write down some basic background on the Grassmann scheme, so that we can exploit the spectrum of $q K_{n: k}$. In Section 3 we prove Theorem 5, and then we finish our investigation with the mentioned consequences in Section 4 and a conclusion in Section 5.

\section{The Grassmann Scheme}

We summarize some needed notation and results for association schemes in the following. Delsarte's PhD thesis [4] provides a deeper introduction into the theory of combinatorial applications of association schemes.

Definition 9. Let $X$ be a finite set. A $k$-class association scheme is a pair $(X, \mathcal{R})$, where $\mathcal{R}=\left\{R_{0}, \ldots R_{k}\right\}$ is a set of symmetric binary relations on $X$ with the following properties:

(a) $\left\{R_{0}, \ldots R_{k}\right\}$ is a partition of $X \times X$.

(b) $R_{0}$ is the identity relation.

(c) There are constants $p_{i j}^{\ell}$ such that for $x, y \in X$ with $(x, y) \in R_{\ell}$ there are exactly $p_{i j}^{\ell}$ elements $z$ with $(x, z) \in R_{i}$ and $(z, y) \in R_{j}$.

Clearly, $\left(X, R_{i}\right)$ is a $p_{0}^{i i}$-regular graph. For convenience, we write $v=|X|$. The relation $R_{i}$ can be described by its adjacency matrix $A_{i}$, so a $(v \times v)-0-1$-matrix which is the indicator function of $R_{i}$. As the $A_{i}$ s are Hermitian and commute, we can diagonalize them simultaneously, that is their eigenvectors are the same. It is well-known that there are $k+1$ common eigenspaces $V_{0}, V_{1}, \ldots, V_{k}$ of the $A_{i}$ s. As the $A_{i}$ s are regular, the all-ones vector $\mathbf{j}$ is an eigenvector and we can assume that $V_{0}=\langle\mathbf{j}\rangle$. Let $E_{i}$ denote the orthogonal projection onto the $i$ th eigenspace. We can express the $A_{i} \mathrm{~s}$ as

$$
A_{i}=\sum_{j=0}^{k} P_{j i} E_{j} .
$$

Note that $P_{0 i}=p_{0}^{i i}$.

The following stability version of Hoffman's bound for independent sets is surely known for a long time. Its first application, at least in the context of intersecting families, which the author is aware of, is due to Ellis [7]. We include a proof, limited to the setting of association schemes, to keep this paper mostly self-contained. 
Lemma 10. Let $\chi$ be the characteristic vector of an independent set of $\left(X, R_{i}\right)$. Assume that $P_{1 i}$ is the smallest eigenvalue of $A_{i}$ and that $P^{-}$is the second smallest eigenvalue of $A_{i}$. Let $E_{r}$ be the orthogonal projection matrix onto the eigenspaces orthogonal to $\langle\mathbf{j}\rangle$ and the eigenspace of $P_{1 i}$. Then

$$
\left(P^{-}-P_{1 i}\right) \chi^{T} E_{r} \chi \leqslant y\left(-P_{1 i}-\frac{P_{0 i}-P_{1 i}}{v} y\right) .
$$

Proof. As $\chi$ is a $0-1$-vector, we have

$$
y=\chi^{T} \chi=\frac{y^{2}}{v}+\sum_{i=1}^{k} \chi^{T} E_{i} \chi .
$$

Hence,

$$
\begin{aligned}
0=\chi^{T} A_{i} \chi & =\frac{P_{0 i}}{v} \chi^{T} \mathbf{J} \chi+\sum_{j=1}^{k} P_{j i} \chi^{T} E_{j} \chi \\
& \geqslant \frac{P_{0 i}}{v} y^{2}+P_{1 i} \sum_{j=1}^{k} \chi^{T} E_{j} \chi+\left(P^{-}-P_{1 i}\right) \chi^{T} E_{r} \chi \\
& \geqslant \frac{P_{0 i}}{v} y^{2}+P_{1 i}\left(y-\frac{y^{2}}{v}\right)+\left(P^{-}-P_{1 i}\right) \chi^{T} E_{r} \chi .
\end{aligned}
$$

Rearranging shows the claim.

The following is surely folklore; see for example the proof of Theorem 2 in [5] for a mostly identical statement.

Lemma 11. Let $\chi$ be the characteristic vector of a non-empty subset $Y$ of $X$, where $y=|Y|$. Let $E_{r}$ be the orthogonal projection matrix onto the eigenspaces orthogonal to $\langle\mathbf{j}\rangle+V_{1}$. Let $P^{-}$be the smallest eigenvalue of $A_{i}$. Then there exists a $T \in Y$ such that at least

$$
\frac{P_{0 i}-P_{1 i}}{v} y+P_{1 i}+\left(P^{-}-P_{1 i}\right) \chi^{T} E_{r} \chi / y
$$

elements of $Y$ are in relation $R_{i}$ to $T$.

Proof. As in the proof of Lemma 10 we obtain

$$
\chi^{T} A_{i} \chi=\frac{P_{0 i}-P_{1 i}}{v} y^{2}+P_{1 i} y+\left(P^{-}-P_{1 i}\right) \chi^{T} E_{r} \chi .
$$

Now averaging shows the claim. 
In the Grassmann scheme $J_{q}(n, k)$ the set of all $k$-spaces of $\mathbb{F}_{q}^{n}$ is $X$ and two subspaces $x$ and $y$ are in relation $R_{i}$ if their intersection is a subspace of dimension $k-i$. Clearly, $R_{k}$ corresponds to adjacency in $q K_{2 k: k}$. The eigenvalues $P_{i j}$ of the Grassmann scheme are well-known. There are two useful formulas, one due to Delsarte [4] and one due to Eisfeld [6]:

$$
\begin{aligned}
& P_{i j}=\sum_{h=0}^{i}(-1)^{i-h} q^{h j+\left(\begin{array}{c}
i-h \\
2
\end{array}\right)}\left[\begin{array}{c}
k-j \\
h
\end{array}\right]\left[\begin{array}{c}
k-h \\
i-h
\end{array}\right]\left[\begin{array}{c}
n-k-j+h \\
h
\end{array}\right] \\
& =\sum_{h=0}^{j}(-1)^{j-h} q^{i(i-j+h)+\left(\begin{array}{c}
j-h \\
2
\end{array}\right)}\left[\begin{array}{l}
j \\
h
\end{array}\right]\left[\begin{array}{c}
k-h \\
i
\end{array}\right]\left[\begin{array}{c}
n-k-j+h \\
n-k-i
\end{array}\right] \text {. }
\end{aligned}
$$

\section{The Weak Hilton-Milner Theorem}

We rely on the following result by Tokushige. Here a pair $(Y, Z), Y, Z \subseteq X$ is a crossintersecting family if all elements in $Y$ intersect all elements of $Z$ non-trivially. Similarly, throughout this section we call an independent set of $q K_{2 k: k}$ an intersecting family.

Theorem 12 (Tokushige (2013)). Let $(Y, Z)$ be a cross-intersecting family of $q K_{n: k}$. Then

$$
|Y| \cdot|Z| \leqslant\left[\begin{array}{l}
n-1 \\
k-1
\end{array}\right]^{2}
$$

For the rest of the section, set $y=\left(1+3 q^{-1}\right)\left[\begin{array}{l}k \\ 1\end{array}\right]\left[\begin{array}{c}2 k-2 \\ k-2\end{array}\right]$. We also assume that $k>3$ as the case $k=3$ was taken care of in [2], and that $Y$ is not a dictator or the dual of a dictator.

\subsection{Proof of Theorem 5}

Lemma 13. Let $\ell$ be a line in $\mathbb{F}_{q}^{n}$. Let $Z$ be a set of $k$-spaces which meet $\ell$ in a fixed point p. Set $Z^{\prime}=\{\langle z, \ell\rangle / \ell: z \in Z\}$. Then $\left|Z^{\prime}\right| \geqslant|Z| /\left[\begin{array}{l}k \\ 1\end{array}\right]$.

Proof. Let $C$ be a complement of $\ell$ in $\mathbb{F}_{q}^{n}$. For $z \in Z$ we have that $\langle z, \ell\rangle$ meets $C$ in a $(k-1)$-space $z^{\prime}$ as $\operatorname{dim}(C)=n-2$ and $\operatorname{dim}(\langle z, \ell\rangle)=k+1$. There are at most $\left[\begin{array}{l}k \\ 1\end{array}\right]$ $k$-spaces $\tilde{z}$ through $p$ in $\left\langle z^{\prime}, \ell\right\rangle$. Hence, at most $\left[\begin{array}{l}k \\ 1\end{array}\right] k$-spaces in $Z$ correspond to the same $(k-1)$-space in $Z^{\prime}$.

Lemma 14. Let $k \geqslant 5$ and $q \geqslant 3$. Let $Y$ be an intersecting family of $q K_{2 k: k}$ of size at least $y$, then no point lies in more than $\left(q^{3-k}\left[\begin{array}{l}k \\ 1\end{array}\right]+1\right)\left[\begin{array}{c}2 k-2 \\ k-2\end{array}\right]$ elements of $Y$.

Proof. First we show that no point $p_{1}$ lies on more than $\left[\begin{array}{l}k \\ 1\end{array}\right]\left[\begin{array}{c}2 k-2 \\ k-2\end{array}\right]$ elements of $Y$. This is clear as otherwise there is a $T \in Y$ with $p_{1} \notin T$. We want to bound the number of $R \in Y$ with $p_{1} \in R$. We have $\left[\begin{array}{l}k \\ 1\end{array}\right]$ choices for one point $p^{\prime}$ in $R \cap T$ and then $\left[\begin{array}{c}2 k-2 \\ k-2\end{array}\right]$ choices for choosing the $k$-space $R$ through $\left\langle p_{1}, p^{\prime}\right\rangle$. Hence, there are at most $\left[\begin{array}{c}k \\ 1\end{array}\right]\left[\begin{array}{c}2 k-2 \\ k-2\end{array}\right]$ elements of $Y$ on $p_{1}$. 
We continue by showing that if one point $p_{1}$ lies in at least $\left(q^{-\alpha}\left[\begin{array}{l}k \\ 1\end{array}\right]+1\right)\left[\begin{array}{c}2 k-2 \\ k-2\end{array}\right]$ elements of $Y$, then all other points lie in at most $\left(1+q^{-1}\right) q^{\alpha+1}\left[\begin{array}{c}2 k-3 \\ k-2\end{array}\right]$ elements of $Y$ which are not on $p_{1}$.

Suppose to the contrary that there are two points $p_{1}$ and $p_{2}$ such that $p_{1}$ lies in at least $\left(q^{-1}\left[\begin{array}{l}k \\ 1\end{array}\right]+1\right)\left[\begin{array}{c}2 k-2 \\ k-2\end{array}\right]$ elements of $Y$. Let $Z_{1}$, respectively, $Z_{2}$ denote the elements of $Y$ on $p_{1}$, respectively, $p_{2}$. Let $\ell$ be $\left\langle p_{1}, p_{2}\right\rangle$. Let $Z_{i}^{\prime}=\left\{\langle\ell, z\rangle / \ell: z \in Z_{i}\right.$ and not $\left.\ell \subseteq z\right\}$ for $i \in\{1,2\}$. Let $x$ be the number of elements of $Y$ containing $\ell$. By Lemma 13, we conclude that $\left|Z_{i}^{\prime}\right| \geqslant\left(\left|Z_{i}\right|-x\right) /\left[\begin{array}{c}k \\ 1\end{array}\right]$ and $\left(Z_{1}, Z_{2}\right)$ is a cross-intersecting family of $(k-1)$-spaces in $\mathbb{F}_{q}^{2 k-2}$. Notice that $x \leqslant\left[\begin{array}{c}2 k-2 \\ k-2\end{array}\right]$, so $\left|Z_{1}\right|-x \geqslant q^{-\alpha}\left[\begin{array}{c}k \\ 1\end{array}\right]\left[\begin{array}{c}2 k-2 \\ k-2\end{array}\right]$. By Theorem 12, we obtain

$$
q^{-\alpha}\left[\begin{array}{l}
k \\
1
\end{array}\right]\left[\begin{array}{c}
2 k-2 \\
k-2
\end{array}\right] \cdot\left(\left|Z_{2}\right|-x\right) \leqslant\left[\begin{array}{c}
k \\
1
\end{array}\right]^{2}\left[\begin{array}{c}
2 k-3 \\
k-2
\end{array}\right]^{2} .
$$

For $q \geqslant 3$, this simplifies to

$$
\left|Z_{2}\right|-x \leqslant q^{\alpha} \frac{q^{k}-1}{q^{2 k-2}-1}\left[\begin{array}{c}
k \\
1
\end{array}\right]\left[\begin{array}{c}
2 k-3 \\
k-2
\end{array}\right] \leqslant\left(1+q^{-1}\right) q^{\alpha+1}\left[\begin{array}{c}
2 k-3 \\
k-2
\end{array}\right]=: b .
$$

Let $R \in Y$. As no point on $R$ except for $p_{1}$ lies in more than $b$ elements of $Y, R$ has $\left[\begin{array}{l}k \\ 1\end{array}\right]$ points and all elements of $Y$ meet $R$ in at least one point, we have

$$
\left(|Y|-\left|Z_{1}\right|\right) /\left(\left[\begin{array}{c}
k \\
1
\end{array}\right]\left[\begin{array}{c}
2 k-2 \\
k-2
\end{array}\right]\right) \leqslant \frac{q^{k}-1}{q^{2 k-2}-1} \cdot\left(1+q^{-1}\right) q^{\alpha+1} \leqslant\left(1+q^{-1}\right) q^{3+\alpha-k} .
$$

Suppose that no point $p_{1}$ lies in at least $q^{-\alpha+1}\left[\begin{array}{l}k \\ 1\end{array}\right]\left[\begin{array}{c}2 k-2 \\ k-2\end{array}\right]>\left(q^{-\alpha}\left[\begin{array}{l}k \\ 1\end{array}\right]+1\right)\left[\begin{array}{c}2 k-2 \\ k-2\end{array}\right]$ elements of $Y$. Then

$$
|Y| /\left(\left[\begin{array}{c}
k \\
1
\end{array}\right]\left[\begin{array}{c}
2 k-2 \\
k-2
\end{array}\right]\right) \leqslant q^{-\alpha+1}+\left(1+q^{-1}\right) q^{3+\alpha-k}
$$

Recall that we can assume that $\alpha \geqslant 1$ as $p_{1}$ lies in at most $\left[\begin{array}{c}k \\ 1\end{array}\right]\left[\begin{array}{c}2 k-2 \\ k-2\end{array}\right]$ elements of $Y$. For $k \geqslant 5$ and $\alpha \geqslant 1$, this is less than $1+3 q^{-1}$, a contradiction as long as $3+\alpha-k \leqslant 0$. Hence, we can choose $\alpha=k-3$ which shows the assertion.

Lemma 15. Let either $k \geqslant 5$ and $q \geqslant 3$, or $k \geqslant 6$ and $q \geqslant 5$. Let $Y$ be an intersecting family of $q K_{2 k: k}$ of size at least $y$. Then there are no points $p_{1}$ and $p_{2}$ such that the number of elements of $Y$ on $p_{1}$ and the number of elements of $Y$ on $p_{2}$ is at least

$$
\left(1-2 q^{3-k}-2 q^{1-k}\right) y /\left[\begin{array}{c}
k-1 \\
1
\end{array}\right]
$$

Proof. Let $Z_{1}$, respectively, $Z_{2}$ denote the elements of $Y$ on $p_{1}$, respectively, $p_{2}$. Set $z_{1}=$ $\left|Z_{1}\right|$ and $z_{2}=\left|Z_{2}\right|$ (we assume $z_{1} \geqslant z_{2}$ ). Suppose that $z_{1}, z_{2} \geqslant\left(1-2 q^{3-k}-2 q^{1-k}\right) y /\left[\begin{array}{c}k-1 \\ 1\end{array}\right]$. Set $\ell=\left\langle p_{1}, p_{2}\right\rangle$. Let $Z_{i}^{\prime}=\left\{\langle\ell, z\rangle / \ell: z \in Z_{i}\right.$ and not $\left.\ell \subseteq z\right\}$ for $i \in\{1,2\}$. As $\ell$ contains at most $\left[\begin{array}{c}2 k-2 \\ k-2\end{array}\right]$ elements of $Y$, we conclude, using Lemma 13 , that $\left|Z_{i}^{\prime}\right| \geqslant\left(z_{i}-\left[\begin{array}{c}2 k-2 \\ k-2\end{array}\right]\right) /\left[\begin{array}{c}k \\ 1\end{array}\right]$. 
As $Y$ is an intersecting family, $\left(Z_{1}, Z_{2}\right)$ is a cross-intersecting family of $(k-1)$-sets in $\mathbb{F}_{q}^{2 k-2}$. By the bound in Theorem 12 , taking the square root and rearranging, we obtain

$$
\left(1-2 q^{3-k}-2 q^{1-k}\right) y /\left[\begin{array}{c}
k-1 \\
1
\end{array}\right]-\left[\begin{array}{c}
2 k-2 \\
k-2
\end{array}\right] \leqslant z_{2}-\left[\begin{array}{c}
2 k-2 \\
k-2
\end{array}\right] \leqslant\left[\begin{array}{c}
k \\
1
\end{array}\right]\left[\begin{array}{c}
2 k-3 \\
k-2
\end{array}\right] .
$$

By using $y=\left(1+3 q^{-1}\right)\left[\begin{array}{l}k \\ 1\end{array}\right]\left[\begin{array}{c}2 k-2 \\ k-2\end{array}\right]$ and rearranging, we obtain

$$
\left(1-2 q^{3-k}-2 q^{1-k}\right)\left(1+3 q^{-1}\right) \leqslant \frac{q^{k-1}-1}{q-1} \cdot \frac{q^{k}-1}{q^{2 k-2}-1}+\frac{q^{k-1}-1}{q^{k}-1} .
$$

This is easily verified to be a contradiction under the conditions on $k$ and $q$.

Lemma 16. Let either $k \geqslant 5$ and $q \geqslant 3$, or $k \geqslant 6$ and $q \geqslant 5$. Let $Y$ be an intersecting family of $q K_{2 k: k}$ of size at least $y$. Let $s$ be the dimension of a smallest subspace meeting all elements of $Y$. Then $s \in\{1, k\}$.

Proof. Let $S$ be a subspace meeting all elements of $Y$. We suppose that $1<\operatorname{dim}(S)<k$ and will arrive at a contradiction, so suppose that $\operatorname{dim}(S)=k-1$ from now on. Let $p_{1}$ and $p_{2}$ the points in $S$ which lie on the most elements of $Y$. Let $Z_{1}$, respectively, $Z_{2}$ denote the elements of $Y$ on $p_{1}$, respectively, $p_{2}$. Set $z_{1}=\left|Z_{1}\right|$ and $z_{2}=\left|Z_{2}\right|$ (we assume $\left.z_{1} \geqslant z_{2}\right)$. By Lemma $14, z_{1} \leqslant\left(q^{3-k}+q^{1-k}\right)\left[\begin{array}{l}k \\ 1\end{array}\right]\left[\begin{array}{c}2 k-2 \\ k-2\end{array}\right]$. Clearly,

$$
z_{2} \geqslant\left(y-z_{1}\right) /\left[\begin{array}{c}
k-1 \\
1
\end{array}\right] \geqslant\left(y-\left(q^{3-k}+q^{1-k}\right)\left[\begin{array}{c}
k \\
1
\end{array}\right]\left[\begin{array}{c}
2 k-2 \\
k-2
\end{array}\right]\right)
$$

Hence, $z_{2} \geqslant\left(1-q^{3-k}-q^{1-k}\right) y /\left[\begin{array}{c}k-1 \\ 1\end{array}\right]$. By Lemma 15 , this is a contradiction. Hence, $s \in\{1, k\}$.

By duality, we obtain the following:

Corollary 17. Let either $k \geqslant 5$ and $q \geqslant 3$, or $k \geqslant 6$ and $q \geqslant 5$. Let $Y$ be an intersecting family of $q K_{2 k: k}$ of size at least $y$. Let $s^{\prime}$ be the dimension of a largest subspace $S$ such that the hyperplanes through $S$ contain all elements of $Y$. Then $s^{\prime} \in\{2 k-1, k\}$.

Hence, in the notation of Lemma 16 and Corollary 17,

$$
\left(s, s^{\prime}\right) \in\{(1,2 k-1),(1, k),(k, 2 k-1),(k, k)\} .
$$

If $s=1$, then all elements of $Y$ lies on a fixed point, so $Y$ is a subset of a dictator. Similarly, if $s=2 k-1$, then all elements of $Y$ lie in a fixed, so is a subset of the dual of a dictator. Hence, we only need to rule out the case $\left(s, s^{\prime}\right)=(k, k)$.

Lemma 18. Let $Y$ be an intersecting family of $q K_{2 k: k}$ of size at least $y$. Then an element in $Y$ meets more than $\left[\begin{array}{l}k \\ 1\end{array}\right]\left[\begin{array}{c}k-1 \\ 1\end{array}\right]$ elements of $Y$ in a $(k-1)$-space. 
Proof. We assume without loss of generality that $y=|Y|$. By Equation (1), we have $P_{0 k}=q^{k^{2}}, P_{1 k}=-q^{k(k-1)}$ as the smallest eigenvalue and $P_{3 k}=-q^{k(k-3)+3}$ as the second smallest eigenvalue of $A_{k}$. By Lemma 10,

$$
\left(P_{3 k}-P_{1 k}\right) \chi^{T} E_{r} \chi \leqslant y\left(-P_{1 k}-\frac{P_{0 k}-P_{1 k}}{v} y\right) .
$$

We have

$$
\begin{aligned}
\frac{-P_{1 k}-\frac{P_{0 k}-P_{1 k}}{v} y}{P_{3 k}-P_{1 k}} & =\frac{q^{k(k-1)}-q^{k^{2}}\left(1+q^{-k}\right) y /\left[\begin{array}{c}
2 k \\
k
\end{array}\right]}{q^{k(k-1)}\left(1-q^{-2 k+3}\right)} \\
& =\frac{1-q^{k}\left(1+q^{-k}\right) y /\left[\begin{array}{c}
2 k \\
k
\end{array}\right]}{1-q^{-2 k+3}} \\
& =\frac{1-q^{k}\left(1+q^{-k}\right)\left(1+3 q^{-1}\right) \frac{\left(q^{k}-1\right)^{2}\left(q^{k-1}-1\right)}{(q-1)\left(q^{2 k}-1\right)\left(q^{2 k-1}-1\right)}}{1-q^{-2 k+3}} \\
& \leqslant \frac{1-q^{-1}\left(1+q^{-k}\right)\left(1+3 q^{-1}\right)\left(1+q^{-1}\right)}{1-q^{-2 k+3}} \leqslant 1-q^{-1}-4 q^{-2} .
\end{aligned}
$$

Hence, $\chi^{T} E_{r} \chi \leqslant y\left(1-q^{-1}-4 q^{-2}\right)$. We want to apply Lemma 11 for $i=1$, so we want to show that

$$
\frac{P_{01}-P_{11}}{v} y+P_{11}\left(q^{-1}+4 q^{-2}\right)+P^{-}\left(1-q^{-1}-4 q^{-2}\right)
$$

is larger than $\left[\begin{array}{c}k-1 \\ 1\end{array}\right]\left[\begin{array}{l}k \\ 1\end{array}\right]$. By Equation (2), $P^{-}=-\left[\begin{array}{l}k \\ 1\end{array}\right], P_{01}=\left[\begin{array}{c}k+1 \\ 1\end{array}\right]\left[\begin{array}{l}k \\ 1\end{array}\right]-\left[\begin{array}{l}k \\ 1\end{array}\right]=q\left[\begin{array}{c}k \\ 1\end{array}\right]^{2}$ and $P_{11}=q\left[\begin{array}{c}k \\ 1\end{array}\right]\left[\begin{array}{c}k-1 \\ 1\end{array}\right]-\left[\begin{array}{l}k \\ 1\end{array}\right]=q^{2}\left[\begin{array}{c}k-2 \\ 1\end{array}\right]\left[\begin{array}{l}k \\ 1\end{array}\right]-1$. Hence, we find

$$
P_{11}\left(q^{-1}+4 q^{-2}\right) \geqslant(q+4)\left[\begin{array}{l}
k \\
1
\end{array}\right]\left[\begin{array}{c}
k-2 \\
1
\end{array}\right]-2 q^{-1} .
$$

and

$$
P^{-}\left(1-q^{-1}-4 q^{-2}\right) \leqslant P^{-} \leqslant\left[\begin{array}{l}
k \\
1
\end{array}\right]
$$

Hence, as $k \geqslant 4, x$ meets at least $(q+3)\left[\begin{array}{l}k \\ 1\end{array}\right]\left[\begin{array}{c}k-2 \\ 1\end{array}\right]$ elements of $Y$ in a $(k-1)$-space. It is easily verified that $(q+3)\left[\begin{array}{c}k-2 \\ 1\end{array}\right]>\left[\begin{array}{c}k-1 \\ 1\end{array}\right]$.

Proof of Theorem 5. As noted before, we only have to rule out that $\left(s, s^{\prime}\right)=(k, k)$ occurs, so suppose that $\left(s, s^{\prime}\right)=(k, k)$. By Lemma 18 , we can find a $k$-space $R^{\prime} \in Y$ which meets more than $\left[\begin{array}{c}k \\ 1\end{array}\right]\left[\begin{array}{c}k-1 \\ 1\end{array}\right]$ elements of $Y$ in a $(k-1)$-space. By averaging over the $\left[\begin{array}{l}k \\ 1\end{array}\right](k-1)$-spaces of $R^{\prime}$, we find a $(k-1)$-space $R$ that lies in more than $\left[\begin{array}{c}k-1 \\ 1\end{array}\right]$ elements of $Y$. As $s=k, R$ is disjoint to one element $T \in Y$. Let $H=\langle R, T\rangle$. Set $Z=\{S \in Y: \operatorname{dim}(S \cap H)=k-1\}$. As there are more than $\left[\begin{array}{c}k-1 \\ 1\end{array}\right]$ elements through $R$, which are all contained in $H$, all 
elements in $Z$ meet $R$ non-trivially. By the dual of Lemma 14, $H$ contains at most $\left(q^{3-k}+q^{1-k}\right)\left[\begin{array}{l}k \\ 1\end{array}\right]\left[\begin{array}{c}2 k-2 \\ k-2\end{array}\right]$ elements of $Y$. Hence,

$$
|Z| \geqslant\left(1-q^{3-k}-q^{1-k}\right) y
$$

By averaging, we find a point $p_{1}$ on at least

$$
z_{1}=\left(1-q^{3-k}-q^{1-k}\right) y /\left[\begin{array}{c}
k-1 \\
1
\end{array}\right]
$$

elements of $Z$ and a point $p_{2}$ in at least

$$
z_{2}=\left(1-2 q^{3-k}-2 q^{1-k}\right) y /\left[\begin{array}{c}
k-1 \\
1
\end{array}\right]
$$

elements of $Z$. By Lemma 15 , this is a contradiction. Hence, $\left(s, s^{\prime}\right)=(k, k)$ does not occur and the proof is complete.

\subsection{Proof of Theorem 6}

Now $y=3\left[\begin{array}{l}k \\ 1\end{array}\right]\left[\begin{array}{c}2 k-2 \\ k-2\end{array}\right]$. For this case the proof is nearly identical to the proof of Theorem 5. Instead of Lemma 14, we just use the crude bound that no point lies on more than $\left[\begin{array}{c}k \\ 1\end{array}\right]\left[\begin{array}{c}2 k-2 \\ k-2\end{array}\right]$ elements of $Y$. The key difference is that we can replace Lemma 15 with the following.

Lemma 19. Let $k \geqslant 4$. Let $Y$ be an intersecting family of $q K_{2 k: k}$ of size at least $y$. Then there are no points $p_{1}$ and $p_{2}$ such that the number of elements of $Y$ on $p_{1}$ or the number of elements of $Y$ on $p_{2}$ is more than

$$
\frac{1}{3} y /\left[\begin{array}{c}
k-1 \\
1
\end{array}\right]
$$

Proof. Our setup is as in the proof of Lemma 15, just that this time the resulting inequality is

$$
\frac{1}{3} y /\left[\begin{array}{c}
k-1 \\
1
\end{array}\right]-\left[\begin{array}{c}
2 k-2 \\
k-2
\end{array}\right] \leqslant z_{2}-\left[\begin{array}{c}
2 k-2 \\
k-2
\end{array}\right] \leqslant\left[\begin{array}{l}
k \\
1
\end{array}\right]\left[\begin{array}{c}
2 k-3 \\
k-2
\end{array}\right] .
$$

By using $y=3\left[\begin{array}{l}k \\ 1\end{array}\right]\left[\begin{array}{c}2 k-2 \\ k-2\end{array}\right]$ and rearranging, we obtain

$$
\frac{q^{k}-1}{q^{k-1}-1} \leqslant 1+\frac{q^{k}-1}{q^{2 k-2}-1} \cdot \frac{q^{k}-1}{q-1}
$$

This is a contradiction. The assertion follows.

From here on it is easy to copy the steps which we took for the proof of Theorem 5, replacing Lemma 14 and Lemma 15 accordingly. 


\section{The Chromatic Number}

In $\left[2\right.$, p. 192] it was established that if $q^{k}+q^{k-1}$ is not the chromatic number and $f$ is the size of the largest independent set which is not contained in a dictator or its dual, then

$$
\left(q^{k}-q^{k-1}\right)\left[\begin{array}{c}
2 k-1 \\
k-1
\end{array}\right] q^{k-1}-f\left[\begin{array}{l}
k \\
1
\end{array}\right]\left[\begin{array}{c}
k+1 \\
1
\end{array}\right]<\epsilon\left(2 f-\left[\begin{array}{c}
2 k-1 \\
k-1
\end{array}\right]\right) .
$$

for some $\epsilon>0$. By Theorem 5,

$$
2 f /\left[\begin{array}{c}
2 k-1 \\
k-1
\end{array}\right] \leqslant 2\left(1+3 q^{-1}\right) \cdot \frac{q^{k}-1}{q-1} \cdot \frac{q^{k-1}-1}{q^{2 k-1}-1} .
$$

For $q \geqslant 5$ this is easily verified to be less than 1 and therefore the right hand side of Equation (3) is negative. Similarly,

$$
\frac{f\left[\begin{array}{c}
k \\
1
\end{array}\right]\left[\begin{array}{c}
k+1 \\
1
\end{array}\right]}{\left(q^{k}-q^{k-1}\right)\left[\begin{array}{c}
2 k-1 \\
k-1
\end{array}\right] q^{k-1}} \leqslant \frac{q^{k-1}-1}{q^{2 k-1}-1} \cdot \frac{\left(q^{k}-1\right)^{2}\left(q^{k+1}-1\right)}{\left(q^{2 k-1}-q^{k-1}\right)(q-1)^{3}}<1,
$$

so the left hand side of Equation (3) is positive. As this is a contradiction, we have shown Theorem 3. Note that [2, Proposition 5.1] gives a characterization of the case of equality.

Theorem 8 is a simple consequence of replacing Theorem 4 with Theorem 5 in the proof of Theorem 7. See [16] for details.

\section{Future Work}

Clearly, the most urgent open cases are the determination of the chromatic number $q K_{2 k: k}$ for $q=2,3,4$. For $q=3,4$ it is sufficient to obtain slightly better stability type results which show $f \leqslant\left[\begin{array}{l}k \\ 1\end{array}\right]\left[\begin{array}{c}2 k-2 \\ k-2\end{array}\right]$ as then $2 f<\left[\begin{array}{c}2 k-1 \\ k-1\end{array}\right]$. For $q=2$ the current approach of determining the chromatic number cannot work as there are examples very close in size to a dictator and its dual.

Classical polar spaces are the geometries induced by non-degenerate sesquilinear forms onto $\mathbb{F}_{q}^{n}$. There are currently barely any stability results known for intersecting families of maximals of finite classical polar spaces in literature and there is an interesting diversity of largest families [19], similar to $q K_{2 k: k}$. Results for cross-intersecting families are known for finite classical polar spaces [13], so it might be feasible to determine their chromatic number in a similar fashion.

\section{Acknowledgements}

I would like to thank the anomymous referee and Jozefien D'haeseleer for their comments on an earlier draft of this document. 


\section{References}

[1] A. Blokhuis, A. E. Brouwer, A. Chowdhury, P. Frankl, T. Mussche, B. Patkós, and T. Szőnyi. A Hilton-Milner theorem for vector spaces. Electron. J. Combin., 17(1):\#R71, 2010.

[2] A. Blokhuis, A. E. Brouwer, and T. Szőnyi. On the chromatic number of $q$-Kneser graphs. Des. Codes Cryptogr., 65(3):187-197, 2012.

[3] A. Blokhuis, M. De Boeck, and J. D'haeseleer. Cameron-Liebler sets of $k$-spaces in PG $(n, q)$. Des. Codes Cryptogr., 2018.

[4] P. Delsarte. An algebraic approach to the association schemes of coding theory. Philips Res. Rep. Suppl., (10):vi+97, 1973.

[5] J. Eisfeld. Subsets of association schemes corresponding to eigenvectors of the BoseMesner algebra. Bull. Belg. Math. Soc. Simon Stevin, 5(2-3):265-274, 1998. Finite geometry and combinatorics (Deinze, 1997).

[6] J. Eisfeld. The eigenspaces of the Bose-Mesner algebras of the association schemes corresponding to projective spaces and polar spaces. Des. Codes Cryptogr., 17(13):129-150, 1999.

[7] D. Ellis. A proof of the Cameron-Ku conjecture. J. Lond. Math. Soc. (2), 85(1):165190, 2012.

[8] P. Erdős, C. Ko, and R. Rado. Intersection theorems for systems of finite sets. Quart. J. Math. Oxford Ser. (2), 12:313-320, 1961.

[9] Y. Filmus and F. Ihringer. Boolean degree 1 functions on some classical association schemes. J. Combin. Theory Ser. A, 162:241-270, 2019.

[10] P. Frankl and R. M. Wilson. The Erdös-Ko-Rado theorem for vector spaces. J. Combin. Theory Ser. A, 43(2):228-236, 1986.

[11] A. J. W. Hilton and E. C. Milner. Some intersection theorems for systems of finite sets. Quart. J. Math. Oxford Ser. (2), 18:369-384, 1967.

[12] W. N. Hsieh. Intersection theorems for systems of finite vector spaces. Discrete Math., 12:1-16, 1975.

[13] F. Ihringer. Cross-intersecting Erdős-Ko-Rado sets in finite classical polar spaces. Electron. J. Combin., 22(2), 2015.

[14] M. Kneser. Aufgabe 300. In Jber. Deutsch. Math.-Verein. 58. 1955.

[15] L. Lovász. Kneser's conjecture, chromatic number, and homotopy. J. Combin. Theory Ser. A, 25(3):319-324, 1978.

[16] K .Metsch. A gap result for Cameron-Liebler k-classes. Discrete Math., 340(6):13111318, 2017.

[17] M. W. Newman. Independent Sets and Eigenspaces. PhD thesis, University of Waterloo, Waterloo, Canada, 2004. 
[18] M. Rodgers, L. Storme, and A. Vansweevelt. The Cameron-Liebler $k$-classes in $P G(2 k+1, q)$. Combinatorica, 38(3):739-757.

[19] V. Pepe, L. Storme, and F. Vanhove. Theorems of Erd?s-Ko-Rado type in polar spaces. J. Combin. Theory Ser. A, 118(4):1291-1312.

[20] N. Tokushige. The eigenvalue method for cross $t$-intersecting families. J. Algebraic Combin., 38(3):653-662, 2013. 